Lucrările Seminarului Geografic Dimitrie Cantemir

Vol. 44, October 2017, pp. 15-26

http://dx.doi.org/10.15551/lsgdc.v44i0.02

\title{
Les défis du développement territorial durable pour la formation des aménageurs - urbanistes : quelques questions introductives à partir des innovations du département aménagement et environnement de Polytech'Tours
}

Jean-Paul Carrière ${ }^{1}$

${ }^{1}$ Ecole Polytechnique de l'Université de Tours, France

To cite this article: Carrière, J.-P.. (2017). Les défis du développement territorial durable pour la formation des aménageurs - urbanistes : quelques questions introductives à partir des innovations du département aménagement et environnement de Polytech'Tours. Lucrările Seminarului Geografic Dimitrie Cantemir, Vol. 44, pp. 15-26. DOI: 10.15551/lsgdc.v44i0.02

To link to this article: http://dx.doi.org/10.15551/lsgdc.v44i0.02 


\title{
LES DÉFIS DU DÉVELOPPEMENT TERRITORIAL DURABLE POUR LA FORMATION DES AMÉNAGEURS - URBANISTES : QUELQUES QUESTIONS INTRODUCTIVES A PARTIR DES INNOVATIONS DU DÉPARTEMENT AMÉNAGEMENT ET ENVIRONNEMENT DE POLYTECH'TOURS
}

\begin{abstract}
Jean-Paul Carrière ${ }^{1}$
Résumé: Cet article pose la question de la manière dont la perspective du Développement territorial Durable (DTD) peut être intégrée dans les formations supérieures en Aménagement du territoire et urbanisme, non pas sous forme d'un quelconque enseignement complémentaire, mais de façon transversale et interdisciplinaire. La démonstration prend appui sur l'expérience du Département Aménagement et Environnement de l'Ecole Polytechnique de l'Université de Tours. Celle-ci n'est pas présentée en détail, mais les principes qui fondent son projet pédagogique sont explicités pour, d'une part, repréciser la conception du développement durable des territoires qui en est à la base, et, d'autre part, indiquer les choix essentiels opérés pour inclure le DTD dans la formation des aménageurs et urbanistes.
\end{abstract}

\section{Introduction}

Lors des éditions des années précédentes du colloque Dimitrie Cantemir (CARRIERE, 2014 et 2015), Nous avions été amenés à aborder à plusieurs reprises la question du Développement territorial durable (DTD) soit sous un angle conceptuel et théorique, soit à travers des champs d'application possibles, tels que le tourisme vert ou la requalification des friches urbaines. Mais il est aussi un autre domaine pour lequel le DTD offre de nouvelles perspectives et suscite un renouvellement des approches, qui est celui de la formation des praticiens et professionnels de l'aménagement et de l'urbanisme.

L'aménagement - urbanisme (AU) est aujourd'hui reconnu en France comme une discipline à part entière, ne serait-ce qu'à travers la création en 1992 d'une section du Conseil National des Universités. C'est donc une discipline jeune, qui de ce fait connaît des évolutions fortes ; tout comme au demeurant le champ d'action auquel elle renvoie, le concept-même d'aménagement appliqué au territoire n'étant apparu que dans les années

\footnotetext{
${ }^{1}$ Professeur émérite en aménagement de l'espace et urbanisme, Ancien directeur du Département Aménagement et Environnement De l'Ecole Polytechnique de l'Université de Tours, Chercheur à l’UMR CNRS 7324 CITERES, Université de Tours, Email : carriere@ univ-tours.fr
} 
$1950^{2}$. Celui-ci, rappelons-le, concerne toutes les échelles territoriales et temporelles, tandis que, sur le plan scientifique, il s'inscrit de plein pied dans le champ des sciences de l'action et du projet, du fait d'une dimension praxéologique très directement affirmée. Depuis son émergence, à la fois en tant que champ d'action publique et domaine de réflexion scientifique, l'AU a connu de nombreuses transformations, liées principalement à la multiplication des échelles et des acteurs, qu'ils soient nationaux (les Etats), locaux (les collectivités), internationaux (en particulier l'Union européenne ${ }^{3}$ ). Mais l'AU a connu également de profondes modifications de son contenu du fait de l'émergence de la problématique de la durabilité, et sous l'influence d'approches transdisciplinaires et systémiques induites par le paradigme de la complexité et les travaux d'Edgard Morin.

En tant que champ d'application de ce paradigme de la complexité, l'AU fait appel à des savoirs multiples, et nécessite en conséquence des projets de formation spécifiques, permettant d'articuler des approches globales et spécialisées, qui ne peuvent se réduire à celle de la géographie, même si cette dernière discipline peut prétendre à une antériorité indiscutable en matière d'identification et d'analyse des problèmes posés par l'organisation de l'espace et son rapport avec le fonctionnement des sociétés.

De façon générale, les formations supérieures en AU connaissent, elles aussi, de rapides changements et conservent un réel caractère «expérimental», à l'instar de la formation d'ingénieur en Génie de l'aménagement et de l'environnement dispensée par l'Ecole Polytechnique de l'Université de Tours ${ }^{4}$, dont les innovations nous serviront ici à développer une réflexion sur le rôle que le DTD peut jouer en tant que référentiel et fil conducteur d'un projet pédagogique original, car ce cursus est fondé sur la volonté de former de futurs aménageurs, aptes à concevoir et à mener des opérations d'aménagement à toutes échelles, et pour tout type de territoire, du rural «profond » à la région métropolitaine, dans une perspective de DTD. En effet, il s'agit tout à la fois de former des professionnels de l'aménagement en capacité de répondre à une multiplicité de problèmes extrêmement variés, qui vont de la sauvegarde de l'environnement naturel, au respect de principes d'équité territoriale, en passant par la résolution de questions concrètes, telles que celles de l'accessibilité, de l'organisation des mobilités ou encore de la mise à disposition de tous les habitants d'un habitat de qualité, pour ne citer que quelques exemples.

Mais pour mieux évaluer la portée et les implications d'un tel projet, il nous faut préalablement revenir sur le concept de DTD, avant de s'interroger sur ses implications en matière de projets d'aménagement et d'urbanisme. C'est en précisant les réponses à ces questions, que l'on peut alors expliquer comment et pourquoi intégrer le DTD dans une formation à l'AU, ce qui sera l'objet de la seconde partie de ce texte.

\footnotetext{
${ }^{2}$ Le terme même d'aménagement du territoire n'apparaît en France qu'à l'occasion de la publication en 1950 du Plan National d'Aménagement du Territoire, proposé par le Ministre de la reconstruction de l'époque, M. Claudius Petit.

${ }^{3}$ Même si l'aménagement du territoire ne relève pas directement des compétences de l'Union, celle-ci en est devenue l'un des acteurs, à travers la production de documents stratégiques, ou la mise en œuvre de sa politique de cohésion et le financement d'opérations locales d'aménagement par les Fonds structurels.

4 Pour plus de détails sur cette formation, le lecteur peut se reporter au site suivant: http://polytech.univ-tours.fr/formations/genie-de-l-amenagement-et-de-l-environnement 405799.kjsp?RH=POLYTECH_FR\&RF=1395832563157
} 


\section{Le développement durable des territoires : de quoi parle-t-on ?}

Comme nous avons déjà eu l'occasion de le préciser dans deux ouvrages récents, rédigés à partir de travaux menés dans le cadre de coopérations franco-roumaines (CARRIERE ET AL. 2013, CARRIERE ET AL. 2016), ou dans plusieurs travaux antérieurs menés au Brésil (CARRIERE, 2013), l'apparition du DTD traduit une complexification du débat épistémologique dans le domaine des études et recherches sur le développement et l'aménagement des territoires urbains ou ruraux. Il résulte d'une forme d'hybridation entre deux approches originellement distinctes du développement, et de ce fait peut être qualifié de "proto-concept » (ROCHMAN, 2008) dans la mesure où il n'apparaît pas complétement stabilisé.

\subsection{Le DTD, un «proto-concept »}

Le DTD est aujourd'hui devenu un référentiel incontournable de l'action publique, et même privée, en matière d'aménagement régional ou urbain, car il ne se trouve plus de document stratégique, de schéma d'aménagement ou de projet opérationnel qui ne l'invoque pas. On peut le constater en France comme en Roumanie. Toutefois, force est de constater que ce terme reste parfois utilisé de façon imprécise, et trop souvent réduit à sa seule dimension environnementale. Il n'est pas exagéré de parler d'une trop fréquente " dérive environnementaliste » de l'utilisation du concept, du fait d'une focalisation exclusive sur les aspects écologiques, alors qu'il est multidimensionnel et concerne tout autant les implications économiques et sociales de l'action aménagiste. En fait, le concept de DTD reste le point de départ d'un paradigme toujours en construction, autant dans la pratique que dans la théorie.

Il est issu du rapprochement de deux visions du développement, que l'on ne saurait réduire l'une à l'autre, à savoir en premier lieu celle du développement territorial, et en second lieu celle du développement soutenable ou «durable». Le développement territorial repose sur les nombreuses théories du développement endogène ou encore du development from below que l'on peut analyser comme autant de tentatives de réponse à trois crises simultanées de la fin des années 70 , à savoir celle du développement "par le haut », centralisé, celle des grands pôles urbano-industriels de l'est comme de l'ouest, et enfin celle de «l'Etat-providence » et de sa capacité à aménager le territoire, provoquée par l'essor de la globalisation et de l'amplification des interdépendances à l'échelle mondiale, de l'effondrement du communisme à l'est et de la remise en question du keynesianisme à l'ouest. Le développement durable, on le sait, a été "impulsé » par la montée des préoccupations environnementales, même si comme on l'a dit plus haut, on ne saurait le réduire à la perspective d'un développement respectueux des contraintes écologiques. La fameuse définition qui en fut donnée par le rapport Brundtland, celle " d'un développement qui répond aux besoins du présent sans compromettre la capacité des générations futures à satisfaire leurs propres besoins. » (BRUNDTLAND, 1987), est devenue des plus courantes, et est très riche par ses implications, en particulier en aménagement et urbanisme, sans pour autant être dénuée d'ambiguïté, comme on le verra plus loin. Développement territorial et développement durable sont associés à des concepts et notions différents : $\mathrm{Si}$ le développement territorial mobilise comme notion-clef celle de territoire, défini de façon 
large en tant qu'espace structuré par des acteurs organisés en réseaux, doté de ressources spécifiques et non transférables (B.PECQUEUR,2006 et 2013) et animé par une socioculture locale, qui lui confère son identite ${ }^{5}$, le développement durable s'inscrit dans une perspective diachronique en privilégiant les principes d'équité intergénérationnelle, de précaution et de prudence écologique. L'un et l'autre conduisent à des priorités stratégiques différentes, qui ne sont pas pour autant nécessairement en contradiction. Ainsi l'approche du développement territorial amène-t-elle à donner priorité à la valorisation des ressources locales spécifiques, matérielles ou immatérielles, plutôt que de mobiliser des ressources exogènes importées. Elle privilégie le renforcement des réseaux et toutes les formes de coopération entre acteurs locaux, même si initialement les intérêts de ceux-ci peuvent diverger. Elle incite à veiller au rééquilibrage interne et à la réduction des inégalités intraterritoriales en renforçant les petits centres, en dépit des tendances majeures et incontournables à la métropolisation, et à renforcer les pouvoirs locaux par une décentralisation effective... On reconnaît là les préconisations essentielles des tenants du développement endogène.

Les principes d'action du développement durable visent, quant à eux à articuler les trois piliers du développement, à savoir l'écologie, l'économie et le social, en évitant la dérive environnementaliste évoquée plus haut. Mais au-delà de ces principes généraux, le développement durable nous invite à repenser à la fois les modes de gouvernance et les périmètres de l'action publique, qui ne sont plus en adéquation avec les limites politicoadministratives.

Le rappel, ici extrêmement sommaire, des concepts-clefs et des principes fondamentaux de mise en œuvre de stratégies relevant soit du développement territorial soit du développement durable ${ }^{6}$, nous laisse cependant entrevoir la possibilité d'une synthèse, à travers la territorialisation du développement durable, même si celui-ci est initialement pensé de façon globale, à l'échelle de la planète entière. Cette synthèse paraît possible pour nous amener à une vision unifiée du DTD. Mais elle paraît également être profondément nécessaire, tant les approches traditionnelles du développement local se heurtent à des limites de durabilité environnementale (Pollution, déchets, congestion urbaine, etc...), mais aussi sociale (fragmentation urbaine, inégalités socio-spatiales, pauvreté et exclusion, etc...) et économique (effets des délocalisations, déclins et restructurations sectoriels, etc...).

Dès lors, articuler les deux perspectives nous paraît à la fois possible et nécessaire, en particulier lorsqu'il s'agit de mettre en application des politiques et projets de développement et d'aménagement local, à l'échelle des villes (des projets d'urbanisme) ou des territoires locaux et régionaux (des projets d'aménagement du territoire et de développement local). Plus que de synthèse, c'est d'une possibilité d'hybridation dont il nous faut parler à ce stade du raisonnement, d'une hybridation permise à la fois par les limites et par les points de convergence entre les deux approches. Leur prise en compte simultanée laisse entrevoir une réelle complémentarité : Les limites du développement territorial, essentiellement centré sur l'économique, concernent une forme « d'oubli » de la

\footnotetext{
5 Pour plus de détails sur cette formation, le lecteur peut se reporter au site suivant : http://polytech.univ-tours.fr/formations/genie-de-l-amenagement-et-de environnement405799.kjsp?RH=POLYTECH FR\&RF=1395832563157

${ }^{6}$ Que nous avions eu l'occasion d'expliciter de façon plus approfondie lors de nos interventions précédentes lors des colloques Dimitrie Cantemir des années précédentes.
} 
dimension environnementale des projets, et de la nécessaire «prudence écologique » qui se doit d'être permanente ; alors que l'expérience nous permet de constater quotidiennement la réalité d'atteintes à l'environnement, souvent irréversibles, résultant d'actions d'aménagement et de développement menées sans prospective et sans précaution écologique. Les limites principales du développement durable, quant à elles, concernent de trop fréquentes difficultés d'opérationnalisation et une approche trop exclusivement " environnementaliste » sous l'influence de la deep ecology qui peut conduire à postuler, au moins de façon implicite, mais excessive et sans nuances, un primat de la Nature sur l'Homme....

C'est pourquoi nous considérons comme possible et nécessaire l'hybridation ou la conjugaison du développement territorial et du développement durable en dépassant leurs limites respectives. Cela est possible à travers la mise en œuvre de projets et de politiques publiques de DTD à différentes échelles.

Cette conviction nous conduit à mettre en avant la notion de territoire de projet comme support de la territorialisation du développement durable ou encore du DTD. Un territoire de projet que l'on peut définir de façon tridimensionnelle par ses dimensions structurelle (son organisation spatiale, ses infrastructures physiques, etc.), institutionnelle (sa réalité politico-administrative) et idéelle (les représentations qu'en ont les acteurs et leurs effets structurants). Comme suggéré plus haut, le territoire de projet peut se penser à toutes les échelles infranationales, et aussi bien à celle du «territoire vécu ou perçu » qu'à celle du « territoire construit» pour reprendre la distinction proposée par B. Pecqueur (B. PECQUEUR, 2006).

Certes, l'hybridation des deux démarches, que l'on pourrait assimiler à une « mise en territoire » du développement durable reste une problématique complexe et fortement contrainte du fait à la fois de la multiplicité des thématiques et actions concrètes auxquelles elle peut conduire, de la persistance des conflits d'usage et de la divergence d'intérêts entre acteurs $^{7}$, ainsi que de l'inertie des comportements, etc. C'est pourquoi la transition vers le DTD implique le recours à l'innovation à la fois technique, institutionnelle et sociale (J. FOREST, A.HAMDOUCH, 2014) de façon à permettre la conception collective de nouvelles « règles du jeu » et une action coordonnée des acteurs territoriaux (élus, aménageurs, populations...). Il s'agit donc de mettre l'innovation au cœur du projet d'aménagement et au service de la durabilité territoriale.

\subsection{DTD et projet d'aménagement}

Envisager le projet d'aménagement d'un territoire dans une perspective de DTD aura en conséquence de ce qui précède de nombreuses incidences sur le contenu - même des projets d'aménagement ou d'urbanisme. Selon Nous, les implications principales du DTD en matière d'aménagement - urbanisme peuvent s'énoncer en six propositions :

- Les projets proposés au titre du DTD doivent s'inscrire dans une vision coévolutive de la relation économie - environnement - société

- Ils seront conçus pour ne pas « stresser » le système écologique tout en cherchant à garantir l'équité sociale... il s'agit tout compte fait d'aménager pour

\footnotetext{
${ }^{7}$ On peut penser ici par exemple à la question de l'affectation de la ressource foncière...
} 
toutes les composantes de la société en ménageant nature, biodiversité et environnement lato sensu.

- Les projets doivent avoir pour finalité ultime la création des conditions sociospatiales d'un « mieux vivre ensemble ».

- Leur conception ne peut faire l'économie d'une prise en compte du long terme et de l'équité intergénérationnelle ; façon de dire qu'ils sont sous-tendus par une vision prospective, tout en découlant d'une démarche pro-active.

- De même, ils sont élaborés à partir de démarches systémiques, transversales et multidimentionnelles, car le DTD implique de considérer le périmètre de l'impact du projet bien au-delà des limites de l'espace qui en est le support physique.

- Enfin, comme suggéré plus haut, les projets relevant du DTD procèdent nécessairement d'une innovation multiforme, qui concerne aussi bien la conception et les techniques de planification et d'aménagement, le suivi et la mise en œuvre, que le contenu des projets.

Au total, intégrer le développement durable à la démarche de projet de territoire et promouvoir le DTD reviennent à :

- Utiliser les trois piliers du développement durable comme fils conducteurs du diagnostic territorial et de la conception du projet d'aménagement.

- Créer les conditions d'une véritable gouvernance participative dépassant les logiques sectorielles et hiérarchiques, de façon à impliquer les populations dans l'organisation de leur espace.

- Recourir à des systèmes d'indicateurs territorialisés de durabilité, simples et mesurables, permettant l'aide à la décision. ${ }^{8}$

Or, le respect de ces principes requiert des compétences spécifiques et rétroagit nécessairement sur le contenu de la formation des aménageurs et des urbanistes. C'est précisément ce que nous souhaitons montrer dans la deuxième partie de ce texte, en nous référant à l'expérience tourangelle du Département Aménagement et Environnement de l'Ecole polytechnique de l'Université de Tours.

Toutefois, avant d'en venir à la présentation de cette expérience de formation d'ingénieurs en aménagement et urbanisme, il Nous paraît souhaitable, en guise de conclusion provisoire et de transition, de laisser s'exprimer Vincent Labeyrie, biologiste, ancien professeur de l'université de Tours et fondateur du Centre d'Etudes Supérieures d'Aménagement, l'ex-CESA qui précéda la création du Département Aménagement et Environnement. Le précurseur de l'écodéveloppement et du développement durable que fut Vincent Labeyrie avait coutume de citer Francis Bacon qui dans son Novum organum écrivait déjà en 1620 : «On ne commande à la nature qu'en lui obéissant... ». Trois siècles plus tard, en 1969, Vincent labeyrie ajoutait :

" Il est impossible pour l'Homme d'intervenir dans ces ensembles que sont l'écosphère et les différents écosystèmes sans connaître les lois de l'écologie, c'est-à-dire l'économie des systèmes naturels [...] Tout projet d'aménagement suppose une connaissance

\footnotetext{
${ }^{8}$ Dans le cadre limité de cet article, nous ne développerons pas ce point, qui pourrait à lui seul faire l'objet d'un autre texte.
} 
des systèmes naturels...mais tout aménageur doit tenir compte de la présence de l'Homme ! [...] La survie de 1'humanité dépend aujourd'hui de son aptitude à procéder à un aménagement judicieux des ressources naturelles et à distribuer harmonieusement les activités...»

Ces extraits d'une plaquette remise par V. Labeyrie aux étudiants à leur entrée dans le cursus de formation préfigurent déjà la façon d'envisager la façon d'envisager la formation des aménageurs et des urbanistes dans une perspective du DTD. En toute hypothèse il nous laisse entrevoir la nécessité d'un enseignement qui articule les Sciences de la Nature et les Sciences humaines et sociales, et à tenir compte simultanément des lois de l'écologie et du fonctionnement de la société globale...La citation suivante est encore plus claire à cet égard : « La crise de l'environnement et un aménagement judicieux des différents types d'espaces impliquent le développement parallèle de formations de divers spécialistes, comme les géophysiciens, les hydrologues, les pédologues, les ingénieurs des Ponts et du Génie Rural, les architectes, les psycho-sociologues, les juristes... et d'aménageurs ${ }^{9}$ qui ont, eux, une vision globale de l'ensemble des problèmes, ce qui permet de mieux les situer. » Cette citation de 1973, bien antérieure à la divulgation du concept de durabilité, a le mérite d'énoncer de façon directe les enjeux d'un enseignement spécifique en aménagement, dépassant le cadre étroit des spécialités requises. En d'autres termes, et en s'inspirant des principes énoncés par V. Labeyrie de penser un enseignement articulant théorie, empirie et apprentissage de l'action. Cela implique une démarche authentiquement transdisciplinaire et intégrée, qui croise les sciences de la ville et des espaces habités (géographie, sociologie, architecture, etc.) les sciences de la nature (écologie, pédologie, biologie, etc.), les sciences $\mathrm{du}$ projet et de l'action (Droit, gestion, recherche opérationnelle, etc.), les sciences humaines, économiques et sociales, ainsi que les outils techniques de l'aménagement (informatique, cartographie, DAO, etc.).

C'est en partant de cette conception que l'on peut en arriver à préciser les motifs et le contenu de formations à l'AU qui procèdent de la perspective du DTD.

\section{Pourquoi et comment intégrer le DTD dans la formation des aménageurs et des urbanistes?}

Retenir le DTD comme élément structurant d'une formation à l'aménagement, telle que celle dispensée par l'université de Tours, répond à la nécessité de former des ingénieurs ${ }^{10}$ aptes à concevoir et/ou à conduire la réalisation de projets d'aménagement du territoire ne créant pas d'irréversibilités environnementales, socialement équitables et inclusifs, et économiquement viables. A contrario, on peut avancer qu'aménager l'espace face aux contraintes nouvelles issues du changement climatique, du délitement des solidarités territoriales et du lien social au cœur des cités ou des espaces à plus faible densité, de la persistance des inégalités socio-spatiales, implique de plus en plus une ingénierie adaptée, permettant d'apporter des solutions à des problèmes inscrits dans des contextes et

\footnotetext{
${ }^{9}$ Souligné par Nous

${ }^{10}$ Nous évoquons ici la formation d'ingénieurs, puisqu'il s'agit en l'occurrence d'une formation en d'ingénieurs en Génie de l'aménagement ; mais nos propos pourraient facilement être élargis à tout autre type de formation supérieure ayant pour objectif de former des professionnels de l'aménagement de haut niveau.
} 
systèmes ouverts et complexes, en avenir incertain ; ce qui fait nécessairement appel à un projet de formation ne faisant pas l'économie du DTD.

C'est en cela, et par le respect de telles exigences, que le projet pédagogique peut entrer en résonance avec la demande sociale en matière de durabilité, ou encore répondre aux injonctions d'origine publique à faire du DTD le socle des politiques et actions d'aménagement; injonctions que l'on retrouve dans les documents stratégiques de l'Etat, de collectivités locales, ou encore de l'UE telles qu'énoncées par la « stratégie de Göteborg ${ }^{11}$ ».

Compte tenu de ce qui précède, la prise en compte des principes du DTD requiert un projet pédagogique répondant à des finalités multiples en combinant des modalités diversifiées. La formation à l'AU, ainsi pensée, doit permettre en premier lieu l'acquisition par les étudiants de connaissances sur tout ce qui a trait à l'espace et aux moyens de le réorganiser en fonction de finalités définies par la société civile ou les pouvoirs publics, ce qui suppose la dispense de cours, complétés par des exercices d'application, concernant les thématiques et les disciplines directement impliquées au cours de l'élaboration d'un projet d'aménagement. Comme déjà indiqué plus haut, il s'agit d'apporter aux étudiants les savoirs de base indispensables et relevant de champs disciplinaires multiples.

Mais le projet pédagogique d'une formation à l'aménagement intégrant les principes du DTD ne saurait se réduire à l'accumulation de savoirs diversifiés, relevant de disciplines différentes. La formation doit aussi être pensée pour permettre à l'étudiant d'apprendre à maîtriser le processus de conception d'un projet et de sa mise en application : c'est ce qui explique la place réservée aux ateliers, stages et travaux de terrain. Ainsi à Tours, au cours des trois années de formation (consécutives à deux années d'enseignement supérieur dans une formation scientifique), l'étudiant devra accomplir 36 semaines de stage ou d'atelier " sur le terrain » en réalisant des travaux visant à réaliser le diagnostic des problèmes à résoudre sur un territoire donné, et à y proposer des solutions adaptées, le cas échéant sous forme de scénarios alternatifs.

De plus, le projet pédagogique, très directement liée au vécu professionnel des aménageurs, a aussi pour finalité l'apprentissage du travail en équipe, car la conception et le suivi du projet d'aménagement relèvent d'un travail éminemment collectif : là encore, les ateliers et les stages professionnels encadrés, en petits groupes, pour répondre à la commande d'un organisme privé ou public, sont organisés pour initier les étudiants à la réalité d'une démarche collective de conception et de réalisation de projets.

L'innovation et la recherche constituent une troisième dimension essentielle du projet pédagogique. C'est pourquoi, ce dernier inclut la réalisation d'un travail de recherche sur 5 mois, qui prendra la forme d'un projet de fin d'étude présenté à travers un mémoire, dont le sujet a été initialement proposé par l'équipe de recherche du Laboratoire rattaché au Département $^{12}$. Ce travail d'initiation à et par la recherche permet à l'étudiant tout à la fois de découvrir comment poser une question de recherche, élaborer une méthodologie et analyser avec la distance critique nécessaire un ensemble hétérogène d'informations et de

\footnotetext{
${ }^{11}$ La stratégie de Göteborg, adoptée par le Conseil Européen des 15 et 16 juin 2001 définit les principes et objectifs de l'Union européenne en matière de développement durable

${ }^{12}$ Il s'agit de l'équipe IPAPE, Ingénierie du Projet en Aménagement, Paysage et Environnement du Laboratoire CITERES (Cités, territoires, Environnement et Société), équipe interdisciplinaire réunissant des chercheurs en $\mathrm{AU}$.
} 
données, ce qui répond à un impératif pour tout aménageur de construire ses propositions d'action territoriale innovante avec réflexivité.

Enfin, cette distance critique n'est envisageable que sous la condition d'une connaissance large des pratiques et politiques de l'aménagement. C'est pourquoi le cursus est organisé de façon, à permettre à la quasi-totalité des étudiants d'accomplir une mobilité internationale, que ce soit en Europe ou vers les autres continents. L'objectif est de permettre aux intéressés de découvrir d'autres cultures et pratiques de l'aménagement, de façon à analyser de façon plus distanciée l'approche française de l'aménagement du territoire. En d'autres termes, la formation est tournée vers l'international pour permettre à ses bénéficiaires de comprendre comment peuvent s'appliquer les principes du DTD dans différents contextes institutionnels et culturels, afin d'arriver à un mieux vivre ensemble par l'organisation de l'espace. Cette orientation se concrétise par des échanges régis par des conventions avec des universités étrangères, dans le cadre du programme européen ERASMUS, ou hors de celui-ci (Chine, Inde, Amérique du Nord, Brésil, etc.)

Au total, à travers la conjugaison de ces différentes modalités pédagogiques, il s'agit de permettre à l'étudiant de découvrir dès sa sélection, à l'entrée dans le cursus, tout ce qui peut être constitutif d'une démarche de projet, tout en lui permettant d'acquérir les compétences spécifiques nécessaires à la mise en œuvre de projets relevant du DTD, au niveau régional ou local.

A l'issue d'un tel parcours de formation, il est attendu que les étudiants puissent :

- Utiliser des indicateurs économiques, sociaux, écologiques comme outils de réflexion stratégique et de conception de projets.

- Créer des indicateurs synthétiques de durabilité liés au projet ou à la politique proposée.

- Apprécier le degré de durabilité, mais aussi de faisabilité de leurs projets.

- Evaluer les impacts du projet dans et au-delà de son périmètre.

- Analyser le jeu des acteurs et leurs interactions pour construire la gouvernance territoriale adaptée au projet du territoire, lorsque le contexte local le permet ; ce qui, il est vrai, est loin d'être vérifié en toute circonstance ${ }^{13}$.

- Maîtriser les connaissances, méthodes et compétences requises pour intervenir auprès des collectivités ou des structures spécialisées de l'aménagement.

\section{Conclusion}

De cette expérience singulière de formation de praticiens et/ou de chercheurs en Aménagement et Urbanisme, nous retiendrons en guise de conclusions de portée générale qu'il apparaît effectivement possible de penser un projet de formation fondé sur le principe de l'hybridation des approches « macro » du développement durable et « micro » (ou « méso ») du développement territorial, à l'occasion de l'élaboration et de la conduite de projets en AU.

${ }^{13}$ Le manque de flexibilité des structures politico-administratives et institutions territoriales ne permet pas toujours aux aménageurs et urbanistes d'intervenir de façon effective dans la construction de la gouvernance locale. Il en résulte alors qu'ils n'ont pas de réelle marge de manœuvre pour faire évoluer les conditions de la gouvernance dans un sens plus propice à la démarche de projet. 
Toutefois, Le contenu du DTD, et donc celui de la formation, reste intimement lié au contexte sociétal et culturel. Par conséquent, on ne saurait imaginer une quelconque transposition directe du cursus tourangeau dans un autre cadre national que celui de la France... Si on peut imaginer que cette expérience puisse en inspirer d'autres dans différents pays, cela ne peut se faire qu'au prix d'adaptations qui tiennent compte des réalités nationales et locales. Comme nous l'avons montré à l'occasion de travaux antérieurs, en particulier lors de recherches comparatives menées au Brésil (CARRIERE, 2013) ou en Roumanie, il ne peut y avoir ni unicité des stratégies locales de DTD (non transposables), ni, sur un plan normatif, universalité d'un modèle d'action. Ceci s'applique aussi à la conception et à la mise en œuvre des projets de formation académique et/ou professionnelle. Mais les bonnes, comme les «mauvaises » pratiques au regard des principes de durabilité, peuvent être comparées.

C'est pourquoi le projet de formation se doit aussi d'apprendre aux étudiants à discerner les contre-références, et à éviter de faire du discours sur le développement durable, qui s'appuie trop souvent sur des visions plus idéologiques que scientifiques, un mauvais alibi pour des projets répondant mal aux exigences de l'éthique en aménagement, et conçus plus en fonction d'intérêts particuliers que de l'intérêt général.

\section{Références bibliographiques}

1. Bacon F., 1986. Novum organum, PUF, Paris.

2. Carrière J.-P., 2014. Développement durable et résilience territoriale. Conférence inaugurale du 34ème colloque Dimitrie Cantemir, Université "Alexandru Ioan Cuza" de Iaşi (Roumanie) avec le soutien de l'ASRDLF, 17 -19 octobre 2014.

3. Carrière J.-P., 2016. La requalification des friches urbaines : enjeu et outil des politiques régionales de développement territorial durable, Lucrările Seminarului Geografic "Dimitrie Cantemir" nr. 43, 5-24

4. Carrière J.-P., Hamdouch A., Iațu C., 2016. Développement durable des territoires, Collection Géographie, Economica - Anthropos, Paris.

5. Carrière J-P, Demazière C, Petrea R, Filimon L., 2013. La mise en œuvre du développement territorial durable. Déclinaisons franco-roumaines. Editions L'Harmattan.

6. Carrière J.-P, 2013. Le développement territorial durable au Brésil : diagnostic des potentialités et des obstacles dans les zones rurales des Etats de la Paraïba et de Santa Catarina, in Guillaume Leturcq et al. : Terrains brésiliens : parcours de recherches en sciences sociales, 195-208, Editions L'Harmattan.

7. Forest J., Hamdouch A. (Eds), 2015. Quand l'innovation fait la ville durable, Presses Universitaires et Polytechniques Romandes, Lausanne.

8. Hamdouch A., Depret M.-H., Tanguy C., 2012 Mondialisation et résilience des territoires. Trajectoires, dynamiques d'acteurs et expériences. Presses Universitaires du Québec, coll. Géographie contemporaine, Québec.

9. Hamdouch A., Depret M.-H., Tanguy C., 2013 « Globalisation, innovation, et échelles géographiques de résilience territoriales. » In KLEIN J-L et ROY M, Pour une nouvelle mondialisation. Le défi d'innover, 211-234, Presses Universitaires du Québec, Québec.

10.Pecqueur B., 2006. Le tournant territorial de l'économie globale, Espaces et sociétés, 124(2), 1732. 
11.Pecqueur B., 2013. "Postfordisme, territoire et durabilité : les nouveaux enjeux des politiques de projet territorial ». Carrière J.-P et al., 2013, La mise en ouvre du développement territorial durable. Déclinaisons franco-roumaines, 37-52, Editions L'Harmattan.

12.Rochman J., 2008. Thèse d'aménagement de l'espace et urbanisme, sous dir. J.-P. Carrière : Analyse critique de l'application des principes du développement territorial durable dans les zones rurales marginalisées du Brésil, Université de Tours. 
